\title{
VSI@ESS: Case study for a vibrational spectroscopy instrument at the european spallation source
}

\author{
Marco Zoppi ${ }^{\mathrm{a}}$, Anna Fedrigo, Milva Celli and Daniele Colognesi \\ Consiglio Nazionale delle Ricerche, Istituto dei Sistemi Complessi, 50019 Sesto Fiorentino, Italy
}

\begin{abstract}
Neutron Vibrational Spectroscopy is a well-established experimental technique where elementary excitations at relatively high frequency are detected via inelastic neutron scattering. This technique attracts a high interest in a large fraction of the scientific community in the fields of chemistry, materials science, physics, and biology, since one of its main applications exploits the large incoherent scattering cross section of the proton with respect to all the other elements, whose dynamics can be spectroscopically detected, even if dissolved in very low concentration in materials composed of much heavier atoms. We have proposed a feasibility study for a Vibrational Spectroscopy Instrument (VSI) at the European Spallation Source ESS. Here, we will summarize the preliminary design calculations and the corresponding McStas simulation results for a possible ToF, Inverted Geometry, VSI beamline.
\end{abstract}

\section{Motivations}

Vibrational Spectroscopy (VS) is an experimental technique that is widely used in the innumerable research laboratories worldwide. When the electromagnetic (EM) radiation is used to probe sample properties, this technique is generally much better known as Infrared Absorption (IA) spectroscopy (a first order interaction process between photons and sample), or as Raman Scattering (RS) spectroscopy (a second order interaction process between photons and sample). However, when matter becomes opaque to the EM radiation or, more generally, the inhomogeneity of the material prevents a deep penetration of the photons in the sample, then the drastic reduction of the effective scattering volume makes the optical methods rather inefficient to probe the bulk properties of the sample and other probes, alternative to EM radiation, should be considered.

Thermal neutrons actually possess the aforementioned characteristics. Their penetration depth in dense matter is roughly independent of the atomic composition and, apart from a few selected and well-defined atomic species, it can easily reach several centimetres below surface, in the bulk volume of the sample [1]. In addition, as neutrons interact directly with nuclei, their scattering properties are not subject to selection rules, which affect IA spectroscopy (electric dipole transitions) and RS spectroscopy (electric polarizability transitions).

Thermal neutron spectroscopic techniques, however, cannot be considered as the panacea for every difficult scientific problem. As a matter of fact, given the high cost of neutrons production and instrumentation, their use must be consequently limited to very special problems whose solution cannot be faced by more standard laboratory techniques. Nonetheless, an increasing number of research

${ }^{\mathrm{a}}$ Corresponding author: marco.zoppi@isc.cnr.it activities needs neutrons as the unique probe which is able to give a quantitative answer to well selected problems concerning the atomic structure and dynamics in a large class of relevant materials. For this reasons, the European scientific community is operating to build in the nearfuture a powerful neutron facility, the European Spallation Source (ESS), following similar achievements recently realized in the USA (the SNS neutron spallation source at ORNL) and in Japan (the MLF neutron spallation source at J-PARC).

ESS is planned to be operative in the next decade, with a basic equipment of about twenty neutron instruments. The aim of the present study is to explore the possibility that a VS instrument might be selected in this group.

\section{Basic features of a neutron vibrational spectrometer at ESS}

At present, most of the scientific output from the neutron vibrational spectroscopy instruments that are operating worldwide has been oriented towards the atomic dynamics of hydrogen-containing materials. This general problem finds primary applications in the fields of Chemistry and Material Science (e.g.: Catalysis, Fuel Cells, Hydrogen-bond Systems, Hydrogen-storage Materials, Zeolites, Polymers, MOF's), Biology (e.g.: Proteins, Drug-delivery), Earth Sciences (e.g.: Dynamics of Nanoconfined Water), and Physics (e.g.: Liquid and Solid Hydrogen, Metal Hydrides) [2]. In most cases, the key factor is played by the high value of the proton incoherent cross section and the measured spectra give relevant quantitative information on the so-called Hydrogen-projected Density of Phonon States. This information, in turn, is of great interest since it allows selecting, owing to pertinent dynamic simulation tools, the most likely position of atomic hydrogen among the various possible residence sites. Incidentally, we remind that the coherent cross-section of hydrogen, i.e. that weighting

This is an Open Access article distributed under the terms of the Creative Commons Attribution License 4.0, which permits unrestricted use, distribution, and reproduction in any medium, provided the original work is properly cited. 


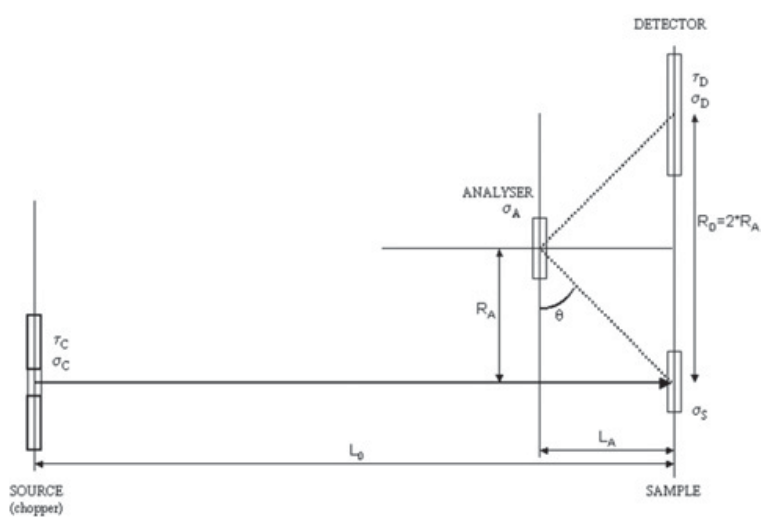

Figure 1. Basic design of an inverted geometry VS-ToF instrument.

the contribution to a neutron diffraction experiment, is extremely small, and therefore the likely position of atomic hydrogen can only be inferred, indirectly, by a residual Fourier maps analysis that is produced by a Rietveld refinement.

\subsection{Inverse geometry basic considerations}

According to Colin Windsor [3], "It is difficult to think of an inelastic neutron spectrometer more versatile than the crystal analyser on a pulsed neutron source". As a matter of fact, the combination of this solution with an inverted geometry design represents the simplest solution for a VS instrument.

The basic instrument design is drawn in Fig. 1. Here the neutron source is defined by the size- and timecharacteristics of the primary pulse. The distance between the source and the sample defines the primary path, $L_{0}$, while the crystal analyser surface plane (in back-scattering configuration) is located at a distance $L_{\mathrm{A}}$ from sample. The nominal Bragg angle, $\theta_{\mathrm{N}}$, is defined by the incidence, on this plane, of the neutron path joining the sample and the analyser centres. The detector plane coincides with the sample one, so to obtain the time focusing condition.

In the inverse geometry design, as schematically depicted in Fig. 1, the wavelength of the scattered neutron (and consequently its energy $E_{1}$ ) is fixed and determined by the Bragg angle on the analyser crystal. This, in turn, determines the Time of Flight (ToF) of the secondary neutron, $t_{1}$. Therefore, knowing the total ToF, measured by the detector, it is possible (by difference) to obtain the primary neutron ToF, $t_{0}$, and from this the energy of the primary neutron, $E_{0}$.

\subsection{Space-time considerations and instrument length}

Ideally, a neutron VS instrument should cover an extremely large energy transfer interval extending from some limited energy gain (anti-Stokes) up to a large energy loss (Stokes), extending up to the range of hydrogen stretching transitions, usually representing the observed peaks of highest energy. As the molecular hydrogen vibrational transition is located at $515 \mathrm{meV}$, a reasonable value for the instrument upper energy limit is set around $500 \mathrm{meV}$.

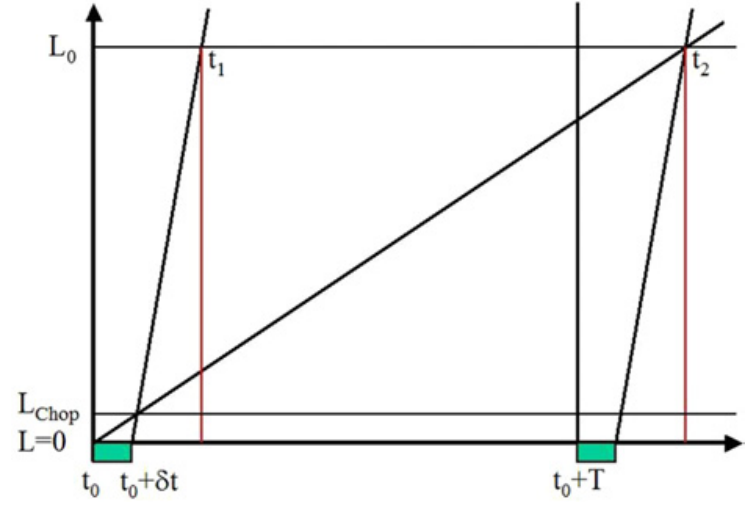

Figure 2. Time-space diagram relative to the preliminary version of the VS instrument. The rectangles (green, online) represent the time-profile of the primary neutrons produced by the moderator; starting at time $t_{0}$ and repeating with the ESS period $T$. Starting from moderator, the sample is located at a distance $L_{0}$ downstream. The fastest neutrons, leaving the moderator at the end of the primary pulse time-window, $\delta t$, reach the sample at time $t_{1}$. Conversely, the slowest neutrons, leaving the moderator at the beginning of the primary pulse time-window, reach the sample at time $t_{2}=t_{1}+T$.

Concerning the energy resolving power, it should be immediately pointed out that a neutron VS instrument will never be able to compete, on this particular aspect, with IA and RS optical instruments. Nevertheless, looking at the corresponding neutron instrumentation available worldwide, it is recognized that a reasonable value for the resolving power should range around $2 \%$, or even better if possible, almost constant in the available energy range.

Let us tentatively assume in the following that the required energy range of the instrument is $500 \mathrm{meV}$. In addition, we also assume that the crystal monochromator is made by pyrolytic graphite and that the selected Bragg angle is set to $45^{\circ}$. As the design frequency for ESS has been set to $14 \mathrm{~Hz}$, the corresponding period turns out to be $T=71.429 \mathrm{~ms}$. In addition, we have to take into account that the ESS design value for the primary pulse timewidth has been set to $\delta t=2.860 \mathrm{~ms}$. Starting from these given values, we will show how we can infer the natural length of the instrument that, in turn, determines the basic parameters for the subsequent Monte Carlo simulations.

If a pyrolytic graphite crystal analyser is used, the interplanar spacing producing the 002 Bragg reflection is $d=3.355 \AA$ [4]. Therefore, a Bragg angle of $45^{\circ}$ selects a neutron wavelength band centred at $\lambda=4.745 \AA$ and corresponding to the energy value $E_{1}=3.633 \mathrm{meV}$. The band width (and integrated intensity) is determined by the graphite mosaic spread. By assuming an instrument energy range extending from the elastic line up to $500 \mathrm{meV}$, we obtain the minimum and the maximum energy values for the incident neutrons. These are: $E_{0, \min }=3.633 \mathrm{meV}$ and $E_{0, \max }=503.633 \mathrm{meV}$, respectively.

The time-space diagram for the preliminary version of the VS instrument is depicted in Fig. 2. As the proposed ESS duty cycle is rather high $(\delta t / T \cong 0.04)$ and represents a serious limitation to the energy resolving power, we should consider inserting a chopper to reduce the time window of the primary pulse, unless we decide to increase 


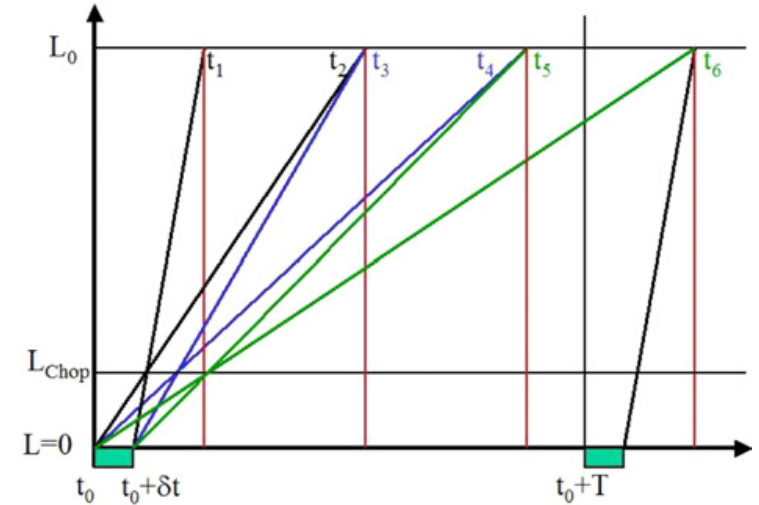

Figure 3. Time-space diagram for the WFM design, using three contiguous time intervals.

the period, by suppressing one or more pulses, with the consequent loss of a consistent fraction of the original neutron flux. The most obvious position for the time shaping chopper corresponds, roughly, to the crossing point of the fast and slow neutron traces in the drawing (cf. Fig. 2).

Once the geometry is fixed, the two parameters $t_{1}$ and $L_{0}$ are univocally determined by the solution of the linear system of equations:

$$
\begin{aligned}
& L_{0}=v_{\min } \cdot t_{2}=v_{\min } \cdot\left(T+t_{1}\right) \\
& L_{0}=v_{\max } \cdot\left(t_{1}-\delta t\right)
\end{aligned}
$$

which can be readily solved providing the unique solution: $t_{1}=9.758 \mathrm{~ms}$ and $L_{0}=67.714 \mathrm{~m}$. The pulse-shaping chopper position turns out to be $L_{\text {chop }}=2.607 \mathrm{~m}$.

However, since the concrete shielding bunkhouse surrounding the target has been designed to extend for a radius of $6 \mathrm{~m}$, at least, we need to move the chopper position downstream. To this aim, we have to resort to the Wavelength Frame Multiplication (WFM) technique [5], using at least three time intervals.

The resulting time-space diagram for the WFM design of the instrument is shown in Fig. 3. Here, we have selected three equal time intervals, which allowed us using a single chopper wheel and three different time-shaping slits. The resulting chopper position is $L_{\text {chop }}=7.262 \mathrm{~m}$ which is sufficiently far away from the moderator and can be placed externally to the concrete shielding bunkhouse.

An interesting feature of this solution is that the three time intervals are contiguous, so that a proper energy can be univocally assigned to each detected neutron. However, since the fast neutrons of each time-interval are faster than the slowest ones of the preceding interval (cf. Fig. 3, where the slopes represent the neutron velocities) we are able, by this design, to obtain some energy superposition of neutrons pertaining to adjacent intervals, which will partially compensate the unavoidable flux decrease produced by the chopper at the boundaries of the selected time intervals.

\section{More detailed design considerations}

Let us now consider that the preliminary parameters we have determined so far, based only on the geometrical configuration, are merely ideal and do not quantitatively correspond to the real experimental situation. In fact, the time shape of the ESS primary pulse is not exactly rectangular but follows the more usual shape, rising from zero to the maximum intensity with a certain time rate, remaining more or less constant at this value for a certain amount of time, and then decreasing to zero with an almost exponential decay rate. In other words, one expects some not negligible amount of neutrons after the nominal end of the primary pulse at time $t_{0}+\delta t$.

The effect of this non-ideal behaviour is totally unwanted, as it causes a contamination of the fast neutrons, produced in the tail of the primary pulse, in the various time intervals. Thus, with reference to Fig. 3, we would expect to have a not negligible amount of fast neutrons, arriving before time $t_{2}$ in the $1^{\text {st }}$ interval, going through the second chopper slit; for the same reason, some fast neutrons will reach the $2^{\text {nd }}$ interval before time $t_{4}$, going through the third chopper slit; finally, fast neutrons will arrive before time $t_{6}$, in the $3^{\text {rd }}$ interval, going through the first chopper slit of the following cycle and produced by the next ESS pulse tail. This fact, among others, would prevent determining, univocally, the energy of each detected neutron from a measure of its ToF. The most obvious solution to this behaviour is to add more choppers to the beamline, to suppress this effect below the detectability level.

\subsection{Chopper system design}

The basic design of a VS instrument at ESS contemplates three choppers. The first is the time shaping chopper, which is characterized by a relatively short time window, to reduce the ToF uncertainty, and by an opening slit sufficiently wide to match the size of the primary beam emerging from the moderator. We calculated a slit angular aperture of $9.25^{\circ}$, assuming a width of $5 \mathrm{~cm}$ for the neutron guide emerging from the concrete shielding bunkhouse and a chopper wheel diameter of $70 \mathrm{~cm}$, which is compatible with the design spatial availability, between neighbouring instruments, at the boundary of the concrete bunkhouse. This choice implies running the chopper at $140 \mathrm{~Hz}$, i.e. 10 times faster than the design frequency of ESS, producing a time window (FWHM) of $183 \mu \mathrm{s}$, which appears reasonable, as far as the instrument resolving power features are concerned.

The wavelength-interval limiting chopper has to be placed downstream, running at the same frequency the first one, so that the same driving engine could be used. For the time being, we have selected this distance to be $\approx 60 \mathrm{~cm}$, corresponding to $\approx 1 \%$ of $L_{0}$. Of course, the phases and the angular openings of this second chopper need to be optimised with regard to the neutron flux and wavelength distribution. Nonetheless, aiming to run a preliminary simulation, we have decided to use the dynamic information based on the results depicted in Fig. 3. This turned out in an opening slit of $\approx 12^{\circ} \mathrm{C}$ corresponding to a time-window of $236 \mu \mathrm{s}$.

Due to the high frequency of the chopper pair running 10 times faster than the ESS frequency, it was necessary to insert a Frame Overlap Chopper to suppress nine out of the ten frames generated by the pair. This was arbitrarily 


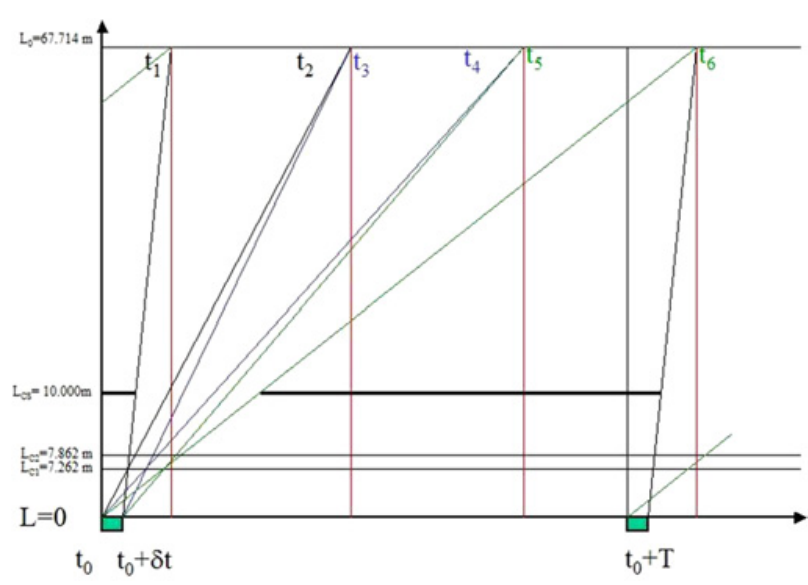

Figure 4. Time-space diagram showing the preliminary choppersystem configuration. The first pair of choppers runs at $140 \mathrm{~Hz}$ and determines the time-window and wavelength extensions of the three selected intervals. The third is a Frame Overlap chopper running at $14 \mathrm{~Hz}$.

inserted at $10 \mathrm{~m}$ distance from the moderator, running at the same frequency of ESS, with an angular aperture of $40.88^{\circ}$. The resulting planned configuration is depicted in Fig. 4.

\subsection{Preliminary simulation results}

The simulation of the primary beamline, as described in the previous sections, was carried out using the McStas simulation package $[6,7]$ including the following components:

1. The ESS water moderator with an output window of $5 \times 8 \mathrm{~cm}^{2}$ at a $6 \mathrm{~m}$ distance.

2. A straight neutron guide $(m=3)$, size $5 \times 8 \mathrm{~cm}^{2}$, delivering the flux to the chopper pair system.

3. A time shaping disk chopper, placed at $7.262 \mathrm{~m}$, disk radius $35 \mathrm{~cm}$, with three angular openings of $9.25^{\circ}$, running at $140 \mathrm{~Hz}$.

4. A wavelength interval selection disk chopper, placed at $7.862 \mathrm{~m}$, disk radius $35 \mathrm{~cm}$, with three angular openings of $11.91^{\circ}$, running at $140 \mathrm{~Hz}$.

5. A straight neutron guide $(m=3)$, size $5 \times 8 \mathrm{~cm}^{2}$, delivering the flux to the next chopper.

6. A frame overlap chopper, placed at $10.000 \mathrm{~m}$, disk radius $35 \mathrm{~cm}$, with one angular opening of $40.88^{\circ}$, running at $14 \mathrm{~Hz}$.

7. A tapering neutron guide $(m=3)$ input size $5 \times$ $8 \mathrm{~cm}^{2}$, output size $4 \times 4 \mathrm{~cm}^{2}$, delivering the flux to the sample position.

8. A collimating window $4 \times 4 \mathrm{~cm}^{2}$, placed at $67.5 \mathrm{~m}$ just before the sample position.

A consistent number of monitors ( $\lambda$, ToF, and XY position sensitive) were strategically placed to check the flux along the beamline. In Fig. 5, we report the results of the detected $\lambda$-distribution in the different positions. From bottom to top, we show the flux detected, respectively:

a) before the chopper system;

b) downstream the first (time shaping) chopper;

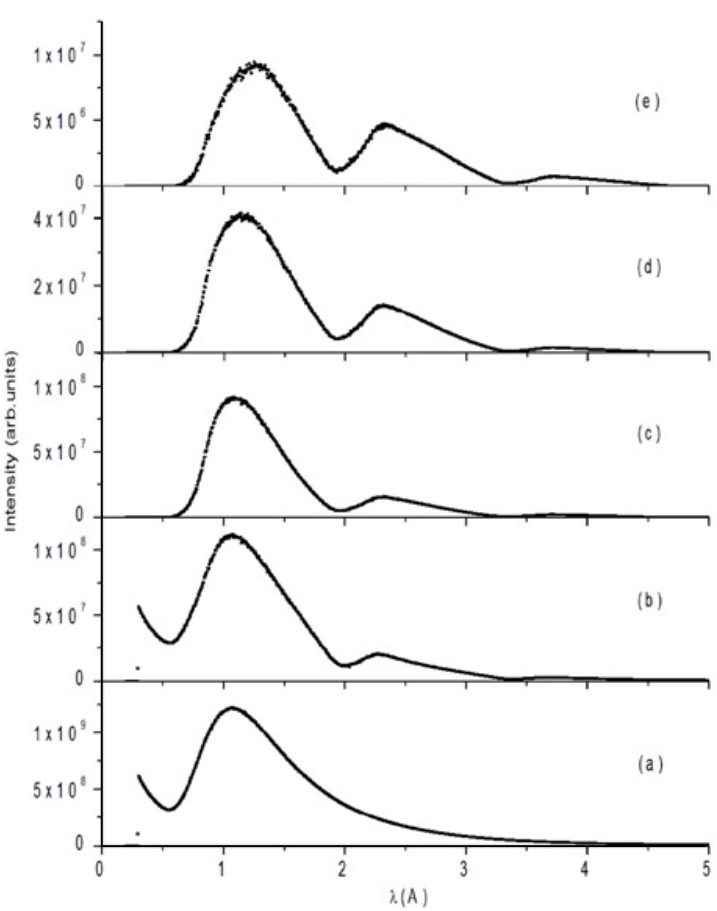

Figure 5. Evolution of the $\lambda$-distribution along the beamline. From bottom to top, we can observe the simulated flux results for: a) before the chopper system; b) downstream the first (time shaping) chopper; c) after the second (wavelength selection) chopper; d) after the third (frame overlap) chopper; e) at sample position.

c) after the second (wavelength selection) chopper;

d) after the third (Frame overlap) chopper;

e) at sample position.

It is worthwhile to note that the effect of the first chopper (time shaping) is simply to reduce the overall neutron flux and to carve away some intensity in the intermediate positions between contiguous chopper openings. The second chopper, instead, cuts out a relevant fraction of the high-energy neutrons and deepens the intensity loss in correspondence with the intervals between two successive chopper apertures. No substantial change is observed after the third (frame overlap) chopper, apart from an overall intensity loss, which appears even more evident for the fast neutrons, owing to the relatively higher losses in the $m=3$ neutron guide. The same effect is also observed in the topmost graph, which is greatly amplified by the much longer path into a tapering neutron guide.

Dealing with the time profile, we will concentrate our attention on the last ToF monitor placed at the sample position, i.e. at $L_{0}=67.714 \mathrm{~m}$, that is shown in Fig. 6. We observe that the intensity does not reach exactly the zero value at $t_{2}=t_{3}=33.57 \mathrm{~ms}$, and at $t_{4}=t_{5}=57.38 \mathrm{~ms}$, as it should be. This implies that the chopper parameters (opening and phase) need to be revised and optimised.

\section{Intensity on sample}

In the previous simulation, we have obtained quantitative indications on the $\lambda$-distribution of neutrons at the sample position. It would be desirable to compare these results 


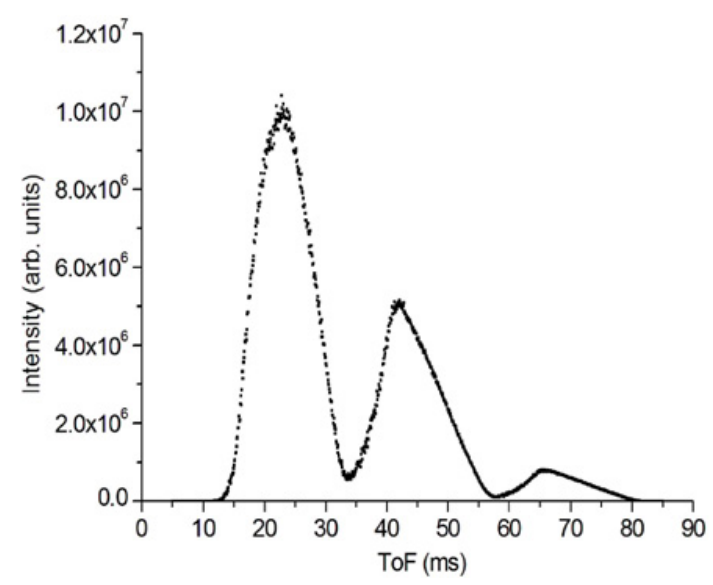

Figure 6. Time dependent flux distribution at sample position. With reference to Fig. 4, the calculated interval time limits are: $t_{1}=9.758 \mathrm{~ms}, t_{2}=t_{3}=33.568 \mathrm{~ms}, t_{4}=t_{5}=57.377 \mathrm{~ms}$, and $t_{6}=81.187 \mathrm{~ms}$.

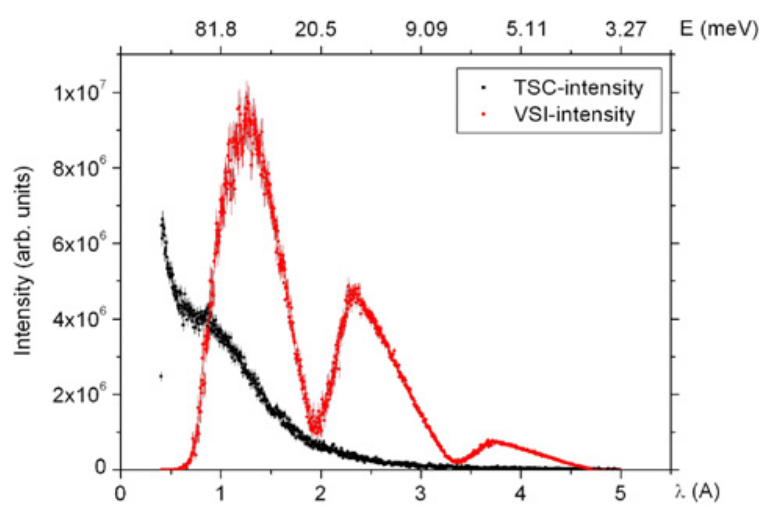

Figure 7. Simulated intensity distributions, at sample position, for the present VS instrument (three-peaks structure, red dots, online) and TOSCA (monotonous shape, black dots). The VSI intensity is the equivalent, as a function of $\lambda$, of that already shown in Fig. 6. The scale on top gives the corresponding energy values.

with the features of a currently working instrument like, for example, TOSCA-II that is operative at ISIS. Therefore, in order to obtain a quantitatively consistent comparison, we have used the McStas package to simulate the primary path of the TOSCA-II spectrometer and to obtain the flux at the sample position. The following components were used for the simulation:

1. The ISIS "TOSCA-type" moderator with an output window of $28 \times 28 \mathrm{~cm}^{2}$ at $1.6 \mathrm{~m}$ distance.

2. Collimating slit, $68 \times 68 \mathrm{~mm}^{2}$ placed at $7.193 \mathrm{~m}$.

3. Collimating slit, $64 \times 64 \mathrm{~mm}^{2}$ placed at $8.410 \mathrm{~m}$.

4. Collimating slit, $62 \times 62 \mathrm{~mm}^{2}$ placed at $8.971 \mathrm{~m}$.

5. Collimating slit, $46 \times 46 \mathrm{~mm}^{2}$ placed at $15.750 \mathrm{~m}$.

6. Sample simulating window, $40 \times 40 \mathrm{~mm}^{2}$ placed at $17.000 \mathrm{~m}$

The monitors ( $\lambda$, ToF, and XY position sensitive) were placed, in sequence, just behind the sample position. In Fig. 7 we show, on the same drawing, the calculated flux at sample position of the TOSCA-II simulation (monotonous shape, black dots) and the VSI simulation (triply-peaked shape, red dots online). We observe that, even in positions of the minima, the VSI flux on sample appears larger than that of TOSCA. However, the consistent lack of intensity in the low-wavelength region and the large width of the two minima call for further work on the chopper system design.

\section{Expected resolving power}

Given the basic instrument design shown in Fig. 1, which basically mimics the TOSCA-II spectrometer at ISIS, it would seem trivial to obtain an analytic evaluation of the expected resolving power applying the same Fortran code used for TOSCA-II after updating the instrument parameters. However, there is basic difference with respect to ISIS, namely the intrinsic time structure of the ESS primary neutron beam and the presence of the time shaping chopper. Thus, we decided to use a different formula that was reported in a recent paper by Seegers et al. [8] to evaluate the expected performances of the VISION spectrometer at SNS.

With reference to Fig. 1, Eq. (21) of Ref. [8] becomes:

$$
\begin{aligned}
& \delta E^{2}=\left(2 E_{0}\right)^{2}\left[\left(\frac{\sigma_{0}}{L_{0}}\right)^{2}+\left(\frac{\sigma_{t}}{t_{0}}\right)^{2}\right. \\
& +\left(\frac{1}{L_{0}}+\frac{1}{v_{A} t_{0}}+\frac{T_{0, A}}{L_{A}}\right)^{2} \sigma_{S}^{2} \\
& \left.+\left(\frac{1}{v_{A} t_{0}}+\frac{T_{0, A}}{L_{A}}\right)^{2} 2 \sigma_{A}^{2}+\left(\frac{1}{v_{A} t_{0}}+\frac{T_{0, A}}{L_{A}}\right)^{2} \sigma_{D}^{2}\right] \\
& +\left(2 E_{A}\right)^{2} \frac{1}{\tan ^{2} \theta}\left(\frac{\sigma_{R A}}{L_{A}}\right)^{2}
\end{aligned}
$$

where $E_{0}$ is the incident neutron energy, $L_{0}$ is primary path (with variance $\sigma_{0}^{2}$ ), and $t_{0}$ is the primary ToF (with variance $\sigma_{t}^{2}$ ). In addition, $\sigma_{S}^{2}$ is the variance associated to the sample thickness, $\sigma_{A}^{2}$ is that relative to the analyser, and $\sigma_{D}^{2}$ pertains to the detector. The two parameters, $v_{A}$ and $E_{A}$, are defined as: $v_{A}=h / 2 m d$ and $E_{A}=\frac{1}{2} m v_{A}^{2}$, and depend only on the neutron mass, $m$, and the crystal analyser plane spacing, $d$; the dimensionless parameter $T_{0, A}$ is defined as:

$$
T_{0, A}=\frac{1}{2} \frac{E_{A}}{E_{0}} \frac{1}{\tan ^{2}(\theta)} .
$$

Finally, $\sigma_{\mathrm{RA}}{ }^{2}$ is the variance associated to the vertical position of the collision point on the graphite crystal (cf. Fig. 1).

In our calculation we have used the same variance values as suggested in Ref. [8], apart from $\sigma_{\mathrm{RA}}{ }^{2}$ that in our case was adapted to a vertical crystal analyser size of $10 \mathrm{~cm}$. It turns out that only two terms in Eq. (2) play a relevant role, while the others give only a negligible contribution. These are: the second term (involving the chopper time shaping window) and the sixth term (involving the vertical size of the crystal analyser). The obtained results are shown in Fig. 8. 


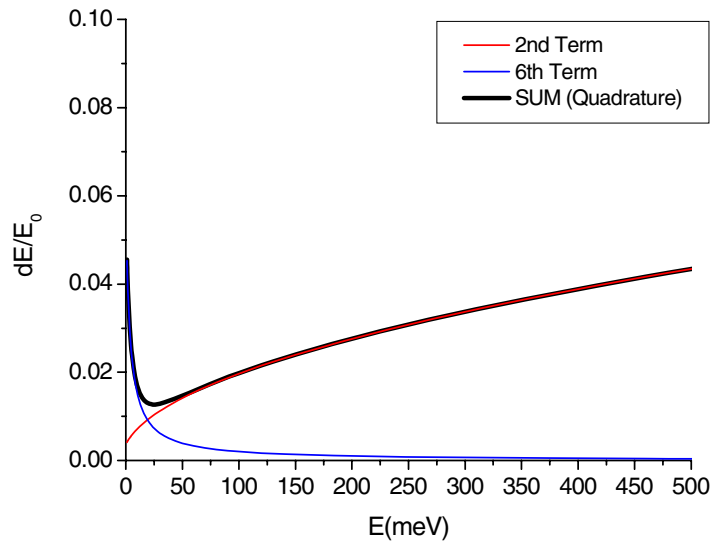

Figure 8. Expected resolving power, calculated according to Eq. (2). Only the $2^{\text {nd }}$ and $6^{\text {th }}$ term give a relevant contribution while the other terms appear almost negligible. The black line represents the sum, in quadrature, of all terms in Eq. (2).

\section{Conclusions}

The main output of this preliminary work is that a VS instrument is feasible on ESS, provided the WFM technique is applied. The resulting instrument length turns out to be of the order of $67 \mathrm{~m}$ and a chopper system is necessary to reduce the time width of the ESS primary pulse. The required characteristics of the chopper system should be arranged in such a way to obtain a net separation of the measured neutron $\mathrm{ToF}$ for the three different intervals in order to fully define the wavelength/energy assignment of each detected neutron. This configuration, however, does not prevent the reconstruction of a continuous wavelength/energy interval extending from the elastic line to the maximum selected energy, which has been temporarily set to $500 \mathrm{meV}$.

The calculated results we have shown, which are relative to the preliminary design described in present work, call for a further tuning of the instrument parameters aiming to optimise the simulated flux at the sample position.

Finally, given the expected instrument length and the relatively short time-window, which is necessary for the proposed VS instrument, we are confident that the obtainable resolving power could be improved, with respect to the existing instrumentation, while the available high luminosity of ESS should be able to provide a sufficient flux to substantially improve also the counting rate.

\section{References}

[1] G. L. Squires, Introduction to the Theory of Thermal Neutron Scattering (Dover, NY, 1996)

[2] P.C.H. Mitchell, S. Parker, A.J. Ramirez Cuesta, J. Tomkinson, Vibrational Spectroscopy with Neutrons (World Scientific, 2005)

[3] C. G. Windsor, Pulsed Neutron Scattering (Taylor \& Francis, London, 1981)

[4] V.K. Pecharsky and P. Y. Zavalij, Fundamentals of Powder Diffraction and Structural Characterization of Materials, $2^{\text {nd }}$ Ed. (Springer, 2009)

[5] F. Mezei, J. Phys. Soc. Jpn. 82, SA025 (2013)

[6] K. Lefmann and K. Nielsen, Neutron News 10/3, 20 (1999)

[7] P. Willendrup, E. Farhi and K. Lefmann, Physica B, 350,735 (2004)

[8] P.A. Seeger, L.L. Daemon, J.Z. Larese, Nucl. Instr. and Meth. A, 604, 719 (2009) 